\title{
Effects of low-molecular-weight heparin and unfractionated heparin on traumatic disseminated intravascular coagulation
}

\author{
Jun-Min Wen ${ }^{1}$, Yu-Xin Sun ${ }^{2}$, Xiao-Hua Pan ${ }^{2}$, Huai-Sheng Chen ${ }^{3 *}$ \\ ${ }^{1}$ Department of Critical Care Medicine, Shenzhen Sun Yat-Sen Cardiovascular Hospital, Shenzhen 518112, ${ }^{2}$ Department of \\ Trauma and Orthopedic, The Second Medical College of Jinan University \& Shenzhen People's Hospital, ${ }^{3}$ Department of \\ Critical Care Medicine, The Second Medical College of Jinan University \& Shenzhen People's Hospital, Shenzhen 518020 , \\ China
}

*For correspondence: Email: jackchendoc@126.com; Tel: +86-0755-25509566

Sent for review: 19 September 2017

Revised accepted: 17 April 2018

\begin{abstract}
Purpose: To explore the effects of unfractionated heparin (UFH) and low-molecular-weight heparin $(L M W H)$ on traumatic disseminated intravascular coagulation (DIC).

Methods: A total of 77 cases of severe trauma (APACHE II score: $5-10$ ) with DIC were collected and randomly assigned to one of three groups: LMWH treatment - 26 cases were subcutaneously injected with LMWH (75-150 units/kg/d); UFH treatment - 25 cases were subcutaneously injected with UFH (100 -250 units/ $/ \mathrm{kg} / \mathrm{d})$; control - 26 cases supplemented with blood coagulation factor only. Daily mortality in the intensive care unit (ICU), hospitalization time, bleeding rate, thrombin time, prothrombin time, activated partial thromboplastin time, and levels of fibrinogen, antithrombin III (ATIII), and D-dimer were recorded and analyzed.

Results: In ICU, LMWH and UFH treatments resulted in lower mortality than in the control group. In addition, hospitalization time was longer in patients treated with LMWH and UFH than in control patients. No significant differences were found between LMWH-treated and control patients in terms of bleeding rate, but UFH-treated patients had lower bleeding rates than control patients. Multifactor analysis indicate a strong relationship between ATIII levels and bleeding rate.

Conclusion: The results indicate that low-dose UFH and LMWH are effective options for the treatment of DIC.
\end{abstract}

Keywords: Trauma, Disseminated intravascular coagulation, Unfractionated heparin, Low-molecularweight heparin, Fibrinogen, Antithrombin

\begin{abstract}
This is an Open Access article that uses a funding model which does not charge readers or their institutions for access and distributed under the terms of the Creative Commons Attribution License (http://creativecommons.org/licenses/by/4.0) and the Budapest Open Access Initiative (http://www.budapestopenaccessinitiative.org/read), which permit unrestricted use, distribution, and reproduction in any medium, provided the original work is properly credited.
\end{abstract}

Tropical Journal of Pharmaceutical Research is indexed by Science Citation Index (SciSearch), Scopus, International Pharmaceutical Abstract, Chemical Abstracts, Embase, Index Copernicus, EBSCO, African Index Medicus, JournalSeek, Journal Citation Reports/Science Edition, Directory of Open Access Journals (DOAJ), African Journal Online, Bioline International, Open-J-Gate and Pharmacy Abstracts

\section{INTRODUCTION}

Disseminated intravascular coagulation (DIC) secondary to severe trauma, infection, and major surgery is a common fatal disease in the intensive care unit (ICU) [1]. At present, other than etiological treatments, hemostatic treatments that supply fibrinogen $(\mathrm{Fg})$, plasma, and blood coagulation factors, as well as plaqueinactivation treatments that involve the infusion of 
unfractionated heparin (UFH), low-molecularweight heparin (LMWH), activated protein $\mathrm{C}$, and antithrombin III (ATIII), are also popular treatments for DIC [2].

However, the most common treatments that use UFH or LMWH are more likely to result in a fatal massive hemorrhage, which makes the practicability and safety of these treatments controversial [3]. Based on the results from a small-scale clinical research study [4], we used low-dose UFH and LMWH to treat patients with traumatic DIC in the ICU from June 2007 to February 2010. In this study, the effects of anticoagulation treatments that alter ATIII levels in the blood were examined.

\section{METHODS}

\section{Subjects}

This research was carried out in accordance with the guidelines of the Declaration of Helsinki [5] and approved by the Medical Ethics Committee of Shenzhen People's Hospital (no. 2006-12$27 \mathrm{Hm}$ ). Patients in the ICU department of our hospital were enrolled in the study from June 2007 to February 2010.

Inclusion criteria were as follows: APACHE II scores between 5 and 10 [6]; diagnosis of acute or chronic DIC; and age greater than 18 years. All enrolled patients or their legal guardians agreed to participate in the research.

Exclusion criteria were as follows: death within $24 \mathrm{~h}$ after a DIC diagnosis; organ hemorrhage; contraindications for heparin therapy; active digestive tract hemorrhage; recent cerebral vascular accident; terminal malignant tumor; massive hemorrhage with dilutional thrombocytopenia; and requirement for emergency surgical care not due to massive hemorrhage.

\section{Experimental protocols}

Patients were diagnosed with DIC using the ISTH scoring scale and evaluated every one or two days. Based on the inclusion and exclusion criteria, 77 patients were included in the study. The patients were divided into three groups as follows: LMWH treatment: 26 cases (mean age, $49 \pm 18.70$ years; APACHEll score, $7.2 \pm 2.6$; DIC score, $3.83 \pm 1.03 ; 54 \%$ male); UFH treatment: 25 cases (mean age, $44.68 \pm 23.8$ years; APACHE II score, $7.8 \pm 2.2$; DIC score, $4.01 \pm 1.11 ; 44 \%$ male); and control: 26 cases treated with coagulation factors only (mean age, $41.7 \pm 19.5$ years; APACHE II score, $7.5 \pm 2.3$;
DIC score, $4.13 \pm 1.52 ; 73 \%$ male). Among the three groups, there were no statistical differences in gender, age, APACHE II score, or DIC score.

All patients were treated using trauma control principles, including the administration of plasma and cryoprecipitates. Patients in the LMWH group were treated with an LMWH infusion, whereas patients in the UFH group were treated with an UFH infusion. The initial dose of LMWH (75 - 150 units $/ \mathrm{kg} / \mathrm{d}$ ) was 4,000 units $/ \mathrm{d}$, and the initial dose of UFH $(100$ - 250 units $/ \mathrm{kg} / \mathrm{d})$ was $5,000 \mathrm{IU} / \mathrm{d}$; the amounts were adjusted based on patients' ATIII levels. When ATIII levels of patients in the LMWH group were lower than $60 \%, 600$ to $800 \mathrm{~mL}$ fresh-frozen plasma were transfused initially, and then 4,000 units LMWH were given by subcutaneous injection twice per day.

When ATIII levels were greater than $60 \%, 4,000$ units LMWH calcium were administered as the primary treatment, and plasma was then transfused, depending on the activated partial thromboplastin time (APTT) and Fg levels. When ATIII levels were greater than $80 \%$, the amount of LMWH administered increased to 4,000 units twice per day. Meanwhile, when ATIII levels of patients in the UFH group were less than $60 \%$, $600-800 \mathrm{~mL}$ fresh-frozen plasma were transfused initially, and 5,000 units UFH were later administered by subcutaneous injection. When ATIII levels were greater than $60 \%$, the amount of infused plasma was determined based on the APTT and Fg levels after transfusing 5,000 units UFH.

When ATIII levels were greater than $80 \%$, the amount of UFH was increased to 10,000 units per day. In the control group, when ATIII levels of patients were lower than $60 \%, 600-800 \mathrm{~mL}$ fresh-frozen plasma were transfused. When ATIII levels were greater than $60 \%$, the amount of plasma was determined based on the APTT and Fg levels. However, once the anticoagulation treatment caused longer APTTs and hemorrhages in patients, the patients in the LMWH group were treated with fresh-frozen plasma, while patients in the UFH group received equal amounts of protamine to counteract the function of the heparin. All treatments were stopped when the thrombin time (TT), prothrombin time (PT), APTT, and Fg levels normalized.

\section{Treatment indices}

In the present study, the following indices were evaluated: 1) DIC score utilizing ISTH guidelines; 2) incidence of death within 28 days, TT, PT, 
APTT, and levels of Fg, ATIII, and D-dimer, and length of hospital stay (two senior ICU doctors concurred on a diagnosis of DIC, the time of death, and discharge from the ICU); 3) hemorrhage rate within 28 days; and 4 ) indices of relative risk $(R R)$, relative risk reduction (RRR), and absolute risk reduction (ARR).

\section{Statistical analysis}

Using SPSS 11.0 software for the analyses, the results are expressed as mean $\pm S D$, and data were compared using analysis of variance. $P<$ 0.05 was considered statistically significant.

\section{RESULTS}

\section{Incidence of death in the three groups}

Five deaths occurred in the LMWH group $(19.2 \%)$, six deaths occurred in the UFH group (24\%), and 15 deaths occurred in the control group $(57.7 \%)$. The relative indices of the LMWH and control groups were as follows: RR = $0.33, \mathrm{RRR}=66.67 \%, \mathrm{ARR}=38.46 \%$, and $X^{2}=$ $6.581(p<0.05)$. The relative indices of the UFH and control groups were as follows: $\mathrm{RR}=0.42$, RRR $=58.40 \%$, ARR $=33.69 \%$, and $X^{2}=4.663$ $(p<0.05)$. The relative indices of the LMWH and UFH groups were as follows: $\mathrm{RR}=0.80, \mathrm{RRR}=$ $19.87 \%$, ARR $=-4.77 \%$, and $X^{2}=0.005(p>$ $0.05)$.

\section{Length of stay in the ICU}

There were statistical differences in the lengths of stay in the ICU among the three groups, as shown by the log-rank analysis method. The $X^{2}$ value was $20.29(p<0.01)$. The area under the Kaplan-Meier curve (Figure 1) revealed that the length of stay in the ICU of the control group was shorter than in both the LMWH and UFH groups, the death incidence was higher $(p<0.01)$, and the length of stay in the ICU of the LMWH group was longer than in the UFH group.

\section{DIC score and PT, APTT, TT, and levels of ATIII, D-dimer, and Fg}

The DIC scores and PT, APTT, TT, and levels of ATIII, D-dimer, and Fg are shown in Tables 1 and 2. APTT and TT reflect the range between standard and actual times.

Table 2: PT and Fg levels in the three groups

\begin{tabular}{lcc}
\hline Variable & PT & Fg \\
\hline LMWH & $3.40 \pm 1.37$ & $2.38 \pm 0.61$ \\
UFH & $2.57 \pm 2.28$ & $2.12 \pm 0.72$ \\
Control & $4.96 \pm 4.18$ & $2.19 \pm 1.07$ \\
$P$ value & $<0.05^{\mathrm{a}}$ & $>0.05^{\mathrm{b}}$ \\
\hline aMWH and UFH groups were compared with the \\
control group separately, $p<0.05$. $^{\mathrm{b}}$ LMWH and UFH \\
groups were compared with the control group \\
separately, $p>0.05$.
\end{tabular}

\section{Incidence of hemorrhage}

During the study duration, 16 patients experienced hemorrhages in the LMWH group $(61.5 \%), 8$ patients experienced hemorrhages in the UFH group (32\%), and 22 patients

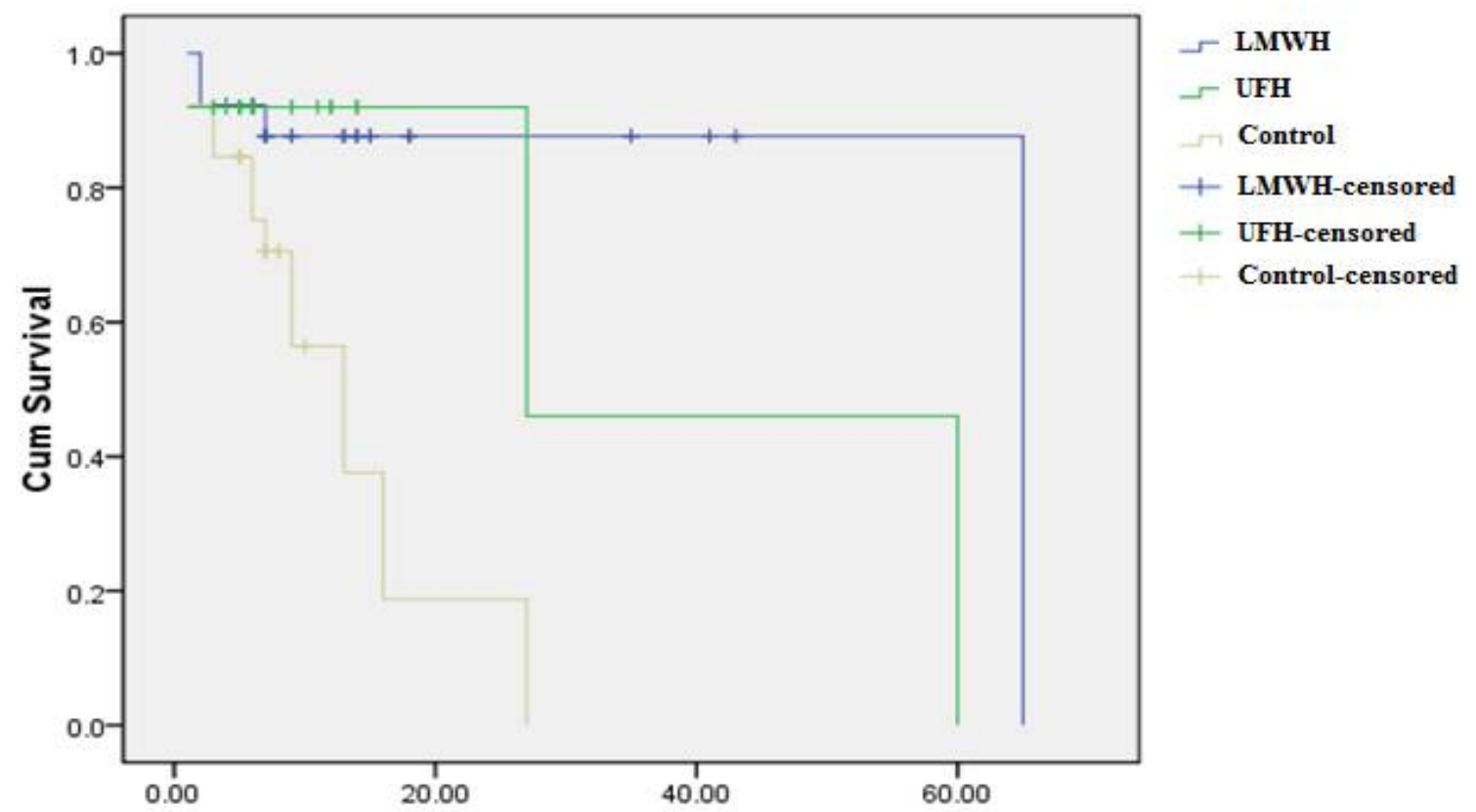

Hospitalization time

Figure 1: Survival analysis curves for the three groups 
Table 1: DIC score for the three groups and APTT, TT, and levels of D-dimer and ATIII

\begin{tabular}{lccccc}
\hline Variable & DIC score & APTT & TT & D-dimer & ATIII \\
\hline LMWH & $3.83 \pm 1.03$ & $6.30 \pm 3.16$ & $3.59 \pm 5.69$ & $6,036.70 \pm 1,943.93$ & $68.98 \pm 16.95$ \\
UFH & $4.01 \pm 1.11$ & $4.63 \pm 2.65$ & $3.94 \pm 4.81$ & $5,151.10 \pm 1,918.47$ & $71.38 \pm 12.10$ \\
Control & $4.13 \pm 1.52$ & $7.23 \pm 5.63$ & $5.22 \pm 5.22$ & $6,155.10 \pm 2,114.58$ & $56.69 \pm 13.39$ \\
Fvalue & 0.68 & 1.32 & 0.80 & 1.32 & 7.79 \\
$P$ value & 0.51 & 0.27 & 0.76 & 0.45 & 0.001 \\
\hline
\end{tabular}

Owing to the heterogeneity of the variance, the Games-Howell analysis method was used to analyze the data

experienced hemorrhages in the control group $(84.6 \%)$. The differences in the incidence of hemorrhage between the control group and the LMWH group, and between the LMWH group and the UFH group, were not statistically significant $\left(X^{2}=3.519, P>0.05\right)$; however, the difference in the incidence of hemorrhage between the UFH group and the control group was statistically significant $\left(X^{2}=14.567, p>\right.$ 0.05). The incidence of hemorrhage in the UFH group was lower than that in the LMWH and control groups, and the difference in the incidence of hemorrhage between the LMWH and control groups were not statistically significant.

\section{Correlation between the incidence of hemorrhage and ATIII levels}

The rate of logistic regression, which assumes that the incidence of hemorrhage is a dependent variable, was $81.3 \%$, with only ATIII levels, PT, and a grouping variable applied to the model. The difference between ATIII levels appears to be statistically significant $(\mathrm{OR}=0.931, p<0.01)$. As the amount of ATIII increased by one unit, the incidence of hemorrhage decreased about $7 \%$. The difference in PTs was not statistically significant, which means that the predictive accuracy of the incidence of hemorrhage was not high.

\section{DISCUSSION}

Direct severe trauma and secondary low perfusion shock are the primary causative factors of DIC [7]. Severe trauma and surgical interventions can induce a coagulation reaction between tissue factors and factor VII that can excessively activate the coagulation pathway and lead to the massive production of coagulation factors. Additionally, the amount of tissue plasminogen released by endothelial cells, stimulated by thrombin after trauma, increases, inhibiting excessive blood clot formation. Overstimulation of the coagulation and fibrinolysis reactions can result in DIC, which has high rates of both hemorrhage and death [8].

To prevent the "death triad" in severe trauma patients (low body temperature, coagulation disorders, and severe acidosis), internal bleeding must be controlled quickly, necrotic tissues must be resected, and traumatic injuries must be controlled according to trauma control principles. Additionally, ICU doctors typically further prevent the death triad by restoring the patient's normal physiological functions [9]. Therefore, anticoagulant treatment should be used to address traumatic DIC. However, technology and patent limitations, abnormal ATIII levels, and activated protein deficiency make UFH and LMWH the most common treatments for DIC and an increased topic of interest in clinical research [10].

The interaction between heparin and ATIII allows heparin to exert its anticoagulant effects: Heparin binds the $\delta$-amino group of a lysine residue in ATIII to accelerate the inactivation of coagulation factors Ila, IXa, Xa, Xia, and XIla, resulting in the inhibition of thrombokinase, XIII activation, and fibrous protein formation. In addition, heparin can also negatively regulate the activation of factors VII and V, which causes platelet aggregation and destruction, inhibiting coagulation [11]. Common heparin is useful in hypercoagulable phages, which continuously reduce the levels of platelets and coagulation factors, as well as the time of microembolization. In consumed hypercoagulable phages, common heparin can be used with coagulation factors to alleviate DIC when the source of the pathogenesis cannot be addressed quickly [12].

In this study, the results showed that ATIII levels decreased by $50 \%$, lowering the effects of heparin and increasing the incidence of hemorrhage, in accordance with results observed in other types of DIC [13]. To overcome these sequelae, studies have begun to focus on LMWH as a treatment. In vitro, LMWH can quickly antagonize factor $\mathrm{X}$ activity during blood clot formation without altering blood coagulation and platelet function. Furthermore, the dose-effect relationship of LMWH is stable because of its low molecular weight and long half-life. Some researchers have demonstrated that the difference between LMWH and UFH is not statistically significant in patients with DIC caused by acute promyelocytic leukemia, suggesting that both have equally curative 
effects [14]. However, LMWH causes a lower incidence of hemorrhage.

The observation time, 28 days, is in accordance with the criteria for assessing fatal DIC $[15,16]$. Based on the specificity and high incidence of death from traumatic DIC in ICU patients, we utilized small doses of LMWH (75 - 150 units $/ \mathrm{kg} / \mathrm{d}$, depending on ATIII levels, with a primary dose of 4,000 units/d) and UFH (100 250 units $/ \mathrm{kg} / \mathrm{d}$, depending on ATIII levels, with a primary dose of $5,000 \mathrm{IU} / \mathrm{d}) \quad,[11,17]$. The incidence of death in the LMWH and UFH groups was lower than that in the control group, and survival time in the ICU was longer in the LMWH and UFH groups than in the control group. Additionally, the UFH group had a shorter discharge time and lower incidence of hemorrhage than the LMWH group, but the death rates were the same between these two groups [18]. Although more large-scale studies are needed, the results of this study showed that low doses of UFH can be used to treat traumatic DIC.

In this study, the differences in ATIII levels among the three groups were significant, as demonstrated by one-way analysis of variance, suggesting that low doses of both UFH and LMWH can increase ATIII levels, regulate the anticoagulation reaction, and decrease the incidences of hemorrhage and death, similar to supplementation with coagulation factors. Related analyses of coagulation factors have also yielded similar results. As ATIII levels decrease, the incidence of hemorrhage increases, which may serve as an indication for adjusting the UFH or LMWH dose to resolve the problem. Therefore, ATIII levels can be used to adjust the dose of heparin. Although the dose of LMWH can be regulated by determining the patient's weight, the results of this study do not show a statistical benefit from the use of LMWH.

\section{CONCLUSION}

Methods based on the trauma control principle (e.g., rescuing supplemental coagulation factors and applying low doses of heparin) help treat DIC. Regulating heparin dose based on ATIII levels can lower the incidences of both hemorrhage and death. In addition, although both the correlation of ATIII levels with the incidence of hemorrhage and the curative effects of the different forms of heparin were not statistically significant, a heparin dose-adjusting strategy may still yield treatment benefits.

\section{DECLARATIONS}

\section{Conflict of Interest}

No conflict of interest associated with this work.

\section{Contribution of Authors}

The authors declare that this work was done by the authors named in this article and all liabilities pertaining to claims relating to the content of this article will be borne by them.

\section{REFERENCES}

1. Levi $M$, Ten Cate $H$. Disseminated intravascular coagulation. N Engl J Med 1999; 341: 586-592.

2. Iba $T$, Kidokoro $A$. Disseminated intravascular coagulation. Nippon Rinsho 2003; 61: 1010-1014.

3. Cornet $A D$, Smit EG, Beishuizen A, Groeneveld $A B$. The role of heparin and allied compounds in the treatment of sepsis. Thromb Haemo 2007; 98: 579-586.

4. Wen JM. Treatment of acute injured disseminated intravascular coagulation with low molecular weight heparin (Fraxiparine) compare with low dose regular heparin. Chin J Thromb Hemo 2005; 11: 116-117.

5. Helsinki Do. The 59th World Medical Association. 2008.

6. Haniffa $R$, Pubudu De Silva A, Weerathunga $P$, Mukaka $M$, Athapattu $P$, Munasinghe $S$, Mahesh $B$, Mahipala $P$, De Silva $T$, et al. Applicability of the APACHE II model to a lower middle income country. J Crit Care 2017; 42:178-183.

7. Wada H, Asakura H, Okamoto K. Expert consensus for the treatment of disseminated intravascular coagulation in Japan. Thromb Res 2010; 125: 6-11.

8. Sawamura A, Hayakawa M, Gando S. Disseminated intravascular coagulation with a fibrinolytic phenotype at an early phase of trauma predicts mortality. Thromb Res 2009; 124: 608-613.

9. Hauser CJ, Holcomb JB, Kluger Y. The coagulopathy of traum: a review of mechanisms. J Trauma 2008; 65: 748-754.

10. CLevi M, Ten C. Disseminated intravascular coagulation. N Engl J Med 1999; 341: 586-592.

11. Cook DJ, Crowther MA. Thromboprophylaxis in the intensive care unit: focus on medical-surgical patients. Crit Care Med 2010; 38: S76-S82.

12. Jin LH, Liu SN, Hu J. Meta-analysis of 65 cases of disseminated intravascular coagulation. Clin Foucs 2009; 24: 1608-1610

13. Tian WH, Wang FC, Huang WZ, Zhong $Y$. The analysis of the effects of heparin in treating 31 cases with disseminated intravascular coagulation. J Southwest Univ Nation 2005; 31:127-128.

14. Hu R, Li $P$, Wang $H$, Liu ZG. Therapeutic effect of heparin and low-molecular-weight heparin (LMWH) on patients with acute promyelocytic leukemia (APL) 
complicated with disseminated intravascular coagulation. Prac Pharm Clin Rem 2007; 10: 83-85

15. Annane D, Bellissant E, Bollaert PE. Corticosteroids in the treatment of severe sepsis and septic shock in adults: a systematic review. JAMA 2009; 301: 23622375.

16. Arós F, Heras M, Vila J. Reduction in 28 days and 6 months of acute myocardial infarction mortality from 1995 to 2005. Rev Esp Cardiol 2011; 64: 972-980.
17. Parikh KC, Oh D, Sittipunt C. Venous thromboembolism prophylaxis in medical ICU patients in Asia (VOICE Asia): a multicenter, observational, cross-sectional study. Thromb Res 2012; 129: 152-158

18. Polderman $K H$, Girbes ARJ. Drug intervention trials in sepsis: divergent results. Lancet 2004; 363: 1721-1723. 